\title{
Discrimination of Myopathy, Neuropathy and Healthy EMG Signals
}

\author{
Amit Kumar Singh ${ }^{1}$, N K Agrawal $^{2}$, Sumit Gupta ${ }^{3}$ \\ Student, Department of Electronics \& Communication, Oriental College of Technology, Bhopal, India ${ }^{1}$ \\ Director, Department of Electronics \& Communication, Oriental College of Technology, Bhopal, India ${ }^{2}$ \\ Associate Professor, Department of Electronics \& Communication, Oriental College of Technology, Bhopal, India ${ }^{3}$
}

\begin{abstract}
In this paper, method for discrimination of EMG signals is proposed. EMG signal is decomposed into set of narrow band signal using empirical mode decomposition (EMD) process known as intrinsic mode functions (IMFs). The features namely mean, standard deviation, variance and Entropy of the intrinsic mode functions (IMFs) generated by EMD process is used to discrimination of myopathy, neuropathy, and healthyEMG signals. The statistical features of IMFs have provided better discrimination performance.
\end{abstract}

Keywords: EMG, EMD, IMF, SVM, RVM.

\section{INTRODUCTION}

Electromyography (EMG) is the process for studying electrical activity produced by muscles. Electrical activities produced by the muscles are collected by using intramuscular electrodes. EMG signals include information about the working and status of muscular system, which makes EMG signal a valuable tool for the diagnosis of neuromuscular disorder such as myopathy and neuropathy [1]. Myopathy is a non-progressive muscular disease, which causes abnormalities, inflammatory, standing, holding things, swallowing, and metabolic disorders [2].

Neuropathy is a neuromuscular rapid progressive disorder, which may affect the working of nerves system in the body. It causes the gradual degeneration of neurons and affects the working of upper and lower motor neurons. Improper functioning of nerves is referred as neuropathy and symptoms includes muscle cramps, weakness, burning, pain, muscle twitching, numbness of the feet or hands [3]. Diagnosis of neuromuscular disorder by visual analysis of EMG signals is difficult, time consuming and may be inaccurate. Due to this, a proper analysis using signals processing may improve the understanding and diagnosis performance of neural diseases. Automatic diagnostic systems can be helpful for the clinicians to detect neuromuscular diseases as well as abnormalities in the neuromuscular system.

Multiscale based features estimated at various scales like instantaneous amplitude, instantaneous frequency and instantaneous phase have been used as input to K-nearest neighbor (KNN), self-organizing map (SOM) and support vector machine (SVM). These classifiers are used for classification of normal and abnormal EMG signals [4]. A combination of mutual information based feature combined with SVM technique has been developed to classify EMG signal [5]. Pattern recognition based strategy has been used for classification of EMG signals [6]. Forearm surface EMG signal has been used for real time control of a robotic arm. Signals produced by muscles in different gestures were recorded which help in controlling prosthetic device with the use of SVM classifier for classification of EMG signals [7]. Real time EMG classification of user-selected intentional forearm movement has been demonstrated for multifunction prosthesis control [8].

\section{METHODOLOGY}

\section{A. Dataset}

The dataset consists signal of a healthy, a neuropathic and a myopathic patient [9]. To collect EMG data from each subject, a $25 \mathrm{~mm}$ needle electrode was used into the tibiali anterior muscle. The location of the needle electrode was changed again and again until motor unit action potentials with a brisk rise time were identified. During the mentioned period of time, the data which was digitized at $50 \mathrm{kHz}$ sampling rate was collected for several seconds and then the patients were asked to relax and needle electrodes were removed. The sampling rate was achieved using a high pass filter and a low-pass filter of sampling rate $20 \mathrm{~Hz}$ and $5 \mathrm{kHz}$ respectively. In this work, 51 subsets of healthy EMG (H) signal, 111 subsets of myopathy EMG signal (M), and 148 subsets of neuropathy EMG signal (N) with each subset of 1000 samples are used for analysis of EMG signals. Fig. 2 shows EMG signals obtained from the dataset. 

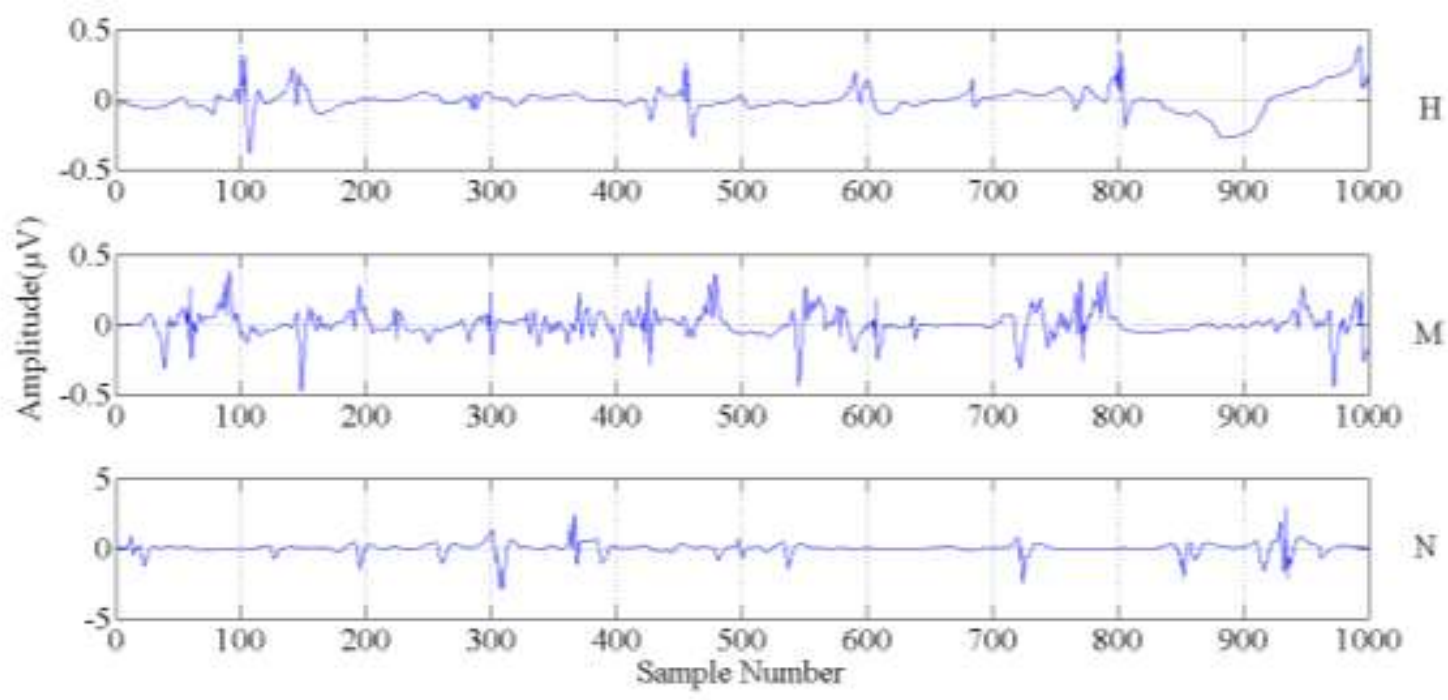

Fig.1 An example of EMG signals from healthy, myopathy and neuropathy EMG signals.

B. Empirical mode decomposition

Empirical mode decomposition is a self-adaptive analysis approach which decomposes non-stationary EMG signal into several intrinsic mode functions (IMFs). EMD does not employ an external basis function for decomposition. EMD has a large number of applications such as audio processing, biomedical, denoising, image processing, fault detection etc. IMF must satisfy two basic conditions [10-11]. Firstly, the number of extrema and number of zero crossing must be equal or differ by at most one. Secondly, the mean value of upper and lower envelope is zero. After decomposition of EMG signal can be represented as:

$$
s(t)=\sum_{m=1}^{M} c_{m}(t)+r_{M}(t)
$$

where, $\$ M \$, \$ c_{-}\{m\}(t) \$$, and $\$ r_{-}\{M\}(t) \$$ denote number of IMF, $\$ m^{\wedge}\{t h\} \$$ IMF and final residue respectively. Recently EMD has been used for analysis and classification of physiological signal [12-14]. IMFs generated by EMD process on healthy, myopathy and neuropathy EMG signal are shown in Fig. 2.
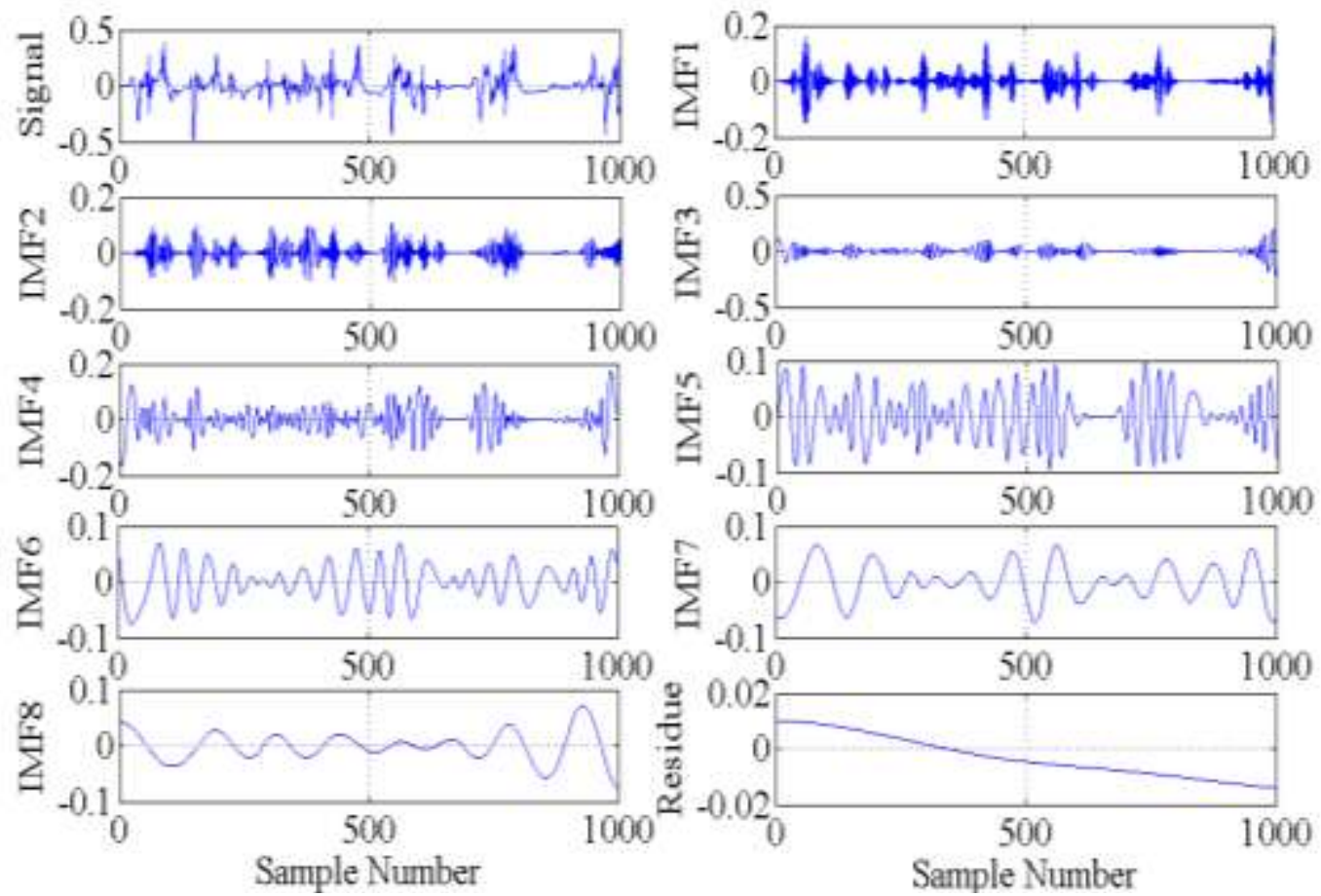

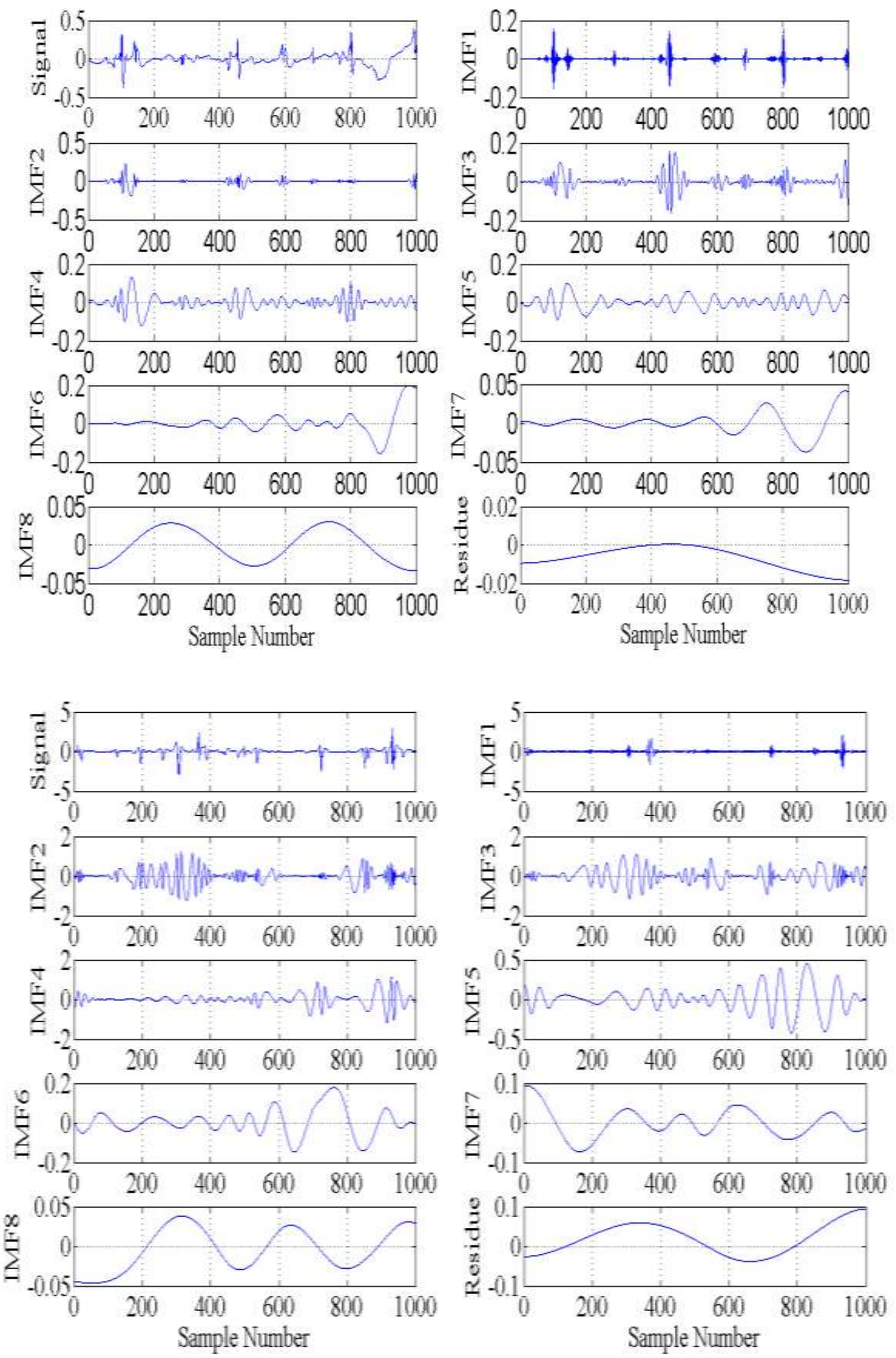

Fig. 2. EMD of healthy, myopathy and neuropathy EMG signals. 
C. Features extracted from IMFs

Emp The statistical features are extracted from IMFs of EMG signals. It can be express as:

Mean: $\mu=\frac{\sum_{i=1}^{N} x_{i}}{N}$

Standard Deviation: $\sigma=\sqrt{\frac{\sum_{i=1}^{N}\left(x_{i}-\mu\right)^{2}}{N}}$

Variance $\sigma=\frac{\sum_{i=1}^{N}\left(x_{i}-\mu\right)^{2}}{N}$

Entropy: $H(x)=\sum_{i=1}^{L} p_{i} \log p_{i}$

\section{III.RESULTS AND DISCUSSION}

EMD decomposes EMG signal into narrow-band AM-FM components (IMFs). Features of the IMFs which has been used as an input to classifier for classification of healthy, myopathy and neuropathy EMG signals. In order to illustrate the effectiveness of features of IMF for time series analysis, the method has been applied to EMG signals. Features of IMFs are computed for classification of myopathy, neuropathy and healthy EMG signals.

Table: 1 Average values of myopathy, neuropathy and healthy EMG signals for first five IMFs

\begin{tabular}{|l|c|l|c|c|c|}
\hline IMFs & Class & Mean & Standard Deviation & Variance & Entropy \\
\hline \multirow{4}{*}{ IMF1 } & healthy & $1.1816^{*} 10^{-4}$ & 0.0175 & $3.2068^{*} 10^{-4}$ & 1.4359 \\
\cline { 2 - 6 } & myopathy & $7.3488^{*} 10^{-4}$ & 0.0305 & 0.0011 & 2.0403 \\
\cline { 2 - 6 } & neuropathy & $7.2123 * 10^{-5}$ & 0.0312 & 0.0011 & 2.0728 \\
\hline \multirow{4}{*}{ IMF2 } & healthy & $-9.7769 * 10^{-4}$ & 0.0363 & 0.0015 & 2.0356 \\
\cline { 2 - 6 } & myopathy & $-6.6485 * 10^{-4}$ & 0.0395 & 0.0017 & 2.3596 \\
\cline { 2 - 6 } & neuropathy & $-6.4872 * 10^{-4}$ & 0.0396 & 0.0017 & 2.3793 \\
\hline \multirow{3}{*}{ IMF3 } & myopathy & $7.0334 * 10^{-4}$ & 0.0345 & 0.0013 & 2.5368 \\
\cline { 2 - 6 } & neuropathy & $5.4627 * 10^{-4}$ & 0.0398 & 0.0017 & 2.7375 \\
\cline { 2 - 6 } & healthy & $5.6378 * 10^{-4}$ & 0.0398 & 0.0017 & 2.7456 \\
\cline { 2 - 6 } & myopathy & $7.2339 * 10^{-5}$ & 0.0315 & 0.0012 & 2.5901 \\
\cline { 2 - 6 } & neuropathy & $1.5397 * 10^{-4}$ & 0.0376 & 0.0016 & 2.7972 \\
\cline { 2 - 6 } & healthy & $1.6229 * 10^{-4}$ & 0.0378 & 0.0016 & 2.8032 \\
\hline
\end{tabular}

\section{IV.CONCLUSION}

We explored the ability of features measurement of intrinsic mode functions for discrimination of myopathy, neuropathy from healthy EMG signals. The proposed method requires selection of input parameters in the algorithm for analysis of EMG signals. Therefore, by choosing the parameters which provides better discrimination, the proposed method may be used as agood dynamical signature to characterize the myopathy and neuropathy in EMG signals. It is illustrate from simulation results that the feature of IMFs is statistically significant for discrimination of healthy, myopathy and neuropathy EMG signals.

\section{REFERENCES}

[1] Hu X, Wang Z, Ren X (2005) Classification of surface EMG signal using relative wavelet packet energy. Computer Methods and Programs in Biomedicine 79(3): 189-195.

[2] Rasheed S, Stashuk D, KamelM(2008) A software package for interactive motor unit potential classification using fuzzy k-NN classifier. Computer Methods and Programs in Biomedicine 89(1): 56-71.

[3] Arabadzhiev TI, Dimitrov GV, Dimitrov AG, Chakarov VE, Dimitrova NA (2008) Factors affecting the turns analysis of the interference EMG signal. Biomedical Signal Processing and Control 3(2): 145-153. 
[4] Christodoulou CI, Kaplanis PA, Murray V, Pattichis MS, Pattichis CS, Kyriakides T (2012) Multi-scale AM-FM analysis for the classification of surface Electromyographic signals. Biomedical Signal Processing and Control 7(3): 265- 269.

[5] Yan Z, Wang Z, Xie H (2008) The application of mutual information-based feature selection and fuzzy LS-SVM-based classifier in motion classification, Computer Methods and Programs in Biomedicine, 90(3): 275-284.

[6] Huang H, Kuiken TA, and Lipschutz RD (2009) A strategy for identifying locomotion modes using surface electromyography, IEEE Transactions on Biomedical Engineering, 56(1): 65-73.

[7] Shenoy P, Miller KJ, Crawford B, Rao RPN (2008) Online electromyography control of a robotic arm. IEEE Transactions on Biomedical Engineering, 55(3): 1128-1135.

[8] http://physionet.org/physiobank/database/emgdb/

[9] Huang NE (1998) et. al, The Empirical mode decomposition and Hilbert spectrum for nonlinear and non-stationary time series analysis. Proc. Roy. Soc. London A, 454: 903-995.

[10] Flandrin P, Rilling G, GoncalvesP(2004) Empirical mode decomposition as a filter bank. IEEE Signal Processing Letters 11: 112-114.

[11] Hassan AR, Haque MA (2016), Computer-aided obstructive sleep apnea identification using statistical features in the EMD domain and extreme learning machine, Biomedical Physics and Engineering Express, 2(3):256-266.

[12] Hassan AR, Bhuiyan MIH (2016), Automatic sleep scoring using statistical features in the EMD domain and ensemble methods Biocybernetics and Biomedical Engineering, 36(1): 248-255

[14] Hassan AR (2015), Automatic screening of obstructive sleepapnea from single-lead electrocardiogram, 2015 International Conference on Electrical Engineering and Information Communication Technology (ICEEICT), 1-6.

\section{BIOGRAPHY}

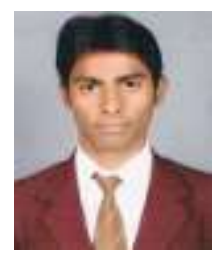

Amit Kumar Singh received the B.E degree in Electronics and communication Engineering from Sagar Institute of Research \& Technology RGPV Bhopal, INDIA in 2014. He is work on NSTEDB project Cell Phone Charging with Voice. He is pursuing M.Tech degree in Electronics and Communication Engineering from Oriental College of Technology RGPV Bhopal INDIA in 2017. He is work on Discrimination of myopathy, neuropathy and healthy EMG signals. 